\section{OP0314 B CELL PHENOTYPE AND FUNCTION IN THE SYNOVIUM OF ACPA+ AND ACPA- RHEUMATOID ARTHRITISPATIENTS}

A. Floudas ${ }^{1}$, M. Biniecka ${ }^{2}$, C. Low ${ }^{2}$, D.J. Veale ${ }^{2}$, U. Fearon ${ }^{1} .{ }^{1}$ Trinity College Dublin; ${ }^{2} U C D$, Dublin, Ireland

Background: Rheumatoid arthritis (RA) is a chronic inflammatory autoimmune disease of unknown and complex etiology with severe detrimental effects for the patient's quality of life. While rheumatoid factors (RF) and anti-citrulinated protein antibodies (ACPA) have been used extensively for the diagnosis of RA, no clear mechanism of action towards disease pathogenesis and progression has been identified. Importantly, both seropositive and seronegative RA patients experience significant improvement in disease severity following $B$ cell depletion. Therefore, we hypothesised that $B$ cells have a central role in $\mathrm{ACPA}^{+}$and $\mathrm{ACPA}^{-} \mathrm{RA}$ irrespective of their capacity to produce auto-antibodies.

Objectives: The characterisation of $B$ and $T$ cell populations in the peripheral blood and synovium of $\mathrm{ACPA}^{+}, \mathrm{ACPA}^{-}$and arthralgia patients.

The identification of non-antibody mediated $B$ cell function under the hypoxic conditions of the inflamed joint.

Methods: Peripheral blood, synovial fluid and tissue was obtained from $\mathrm{ACPA}^{+}$, $\mathrm{ACPA}^{-}$and arthralgia patients. Following enzyme digestion of the tissue, several 15 -colour panels were used for the flow cytometric analysis of $T$ and $B$ cell populations of $\mathrm{ACPA}^{+}, \mathrm{ACPA}^{-}$and arthralgia patients compared to healthy subjects. Activation and function of healthy, sorted $\mathrm{B}$ cells, cultured in vitro and stimulated by CD40 and BCR mediated signals under normoxic $\left(21 \% \mathrm{O}_{2}\right)$ and hypoxic $\left(1 \% \mathrm{O}_{2}\right)$ conditions was examined.

Results: Pro-inflammatory cytokine production by peripheral blood $\mathrm{CD} 4^{+} \mathrm{T}$ cells is not significantly different between $\mathrm{ACPA}^{+}, \mathrm{ACPA}^{-}$and arthralgia patients when compared to healthy controls. However, a significant reduction in $\mathrm{CD} 27^{+}$switched memory B cells was observed between healthy subjects and $\mathrm{APCA}^{+} \mathrm{RA}$ patients. The aforementioned decrease in memory $B$ cells is potentially a result of increased susceptibility to FAS induced apoptosis since healthy B cells cultured with RA patient plasma showed increased activation, CD80/CD86 and FAS expression.

In the synovial fluid and synovial tissue, CD4 T cell pro-inflammatory cytokine production was increased when compared to peripheral blood CD4 T cells. Interestingly, ACPA RA patient $C D 4^{+} \mathrm{T}$ cells produced reduced amounts of proinflammatory cytokines when compared to $\mathrm{ACPA}^{+} \mathrm{RA}$ patient $\mathrm{CD4} 4^{+} \mathrm{T}$ cells. Despite accumulation of switched and double negative (DN) memory $B$ cells in the synovial fluid and tissue, compared to peripheral blood, no differences in synovial $\mathrm{B}$ cell subpopulation composition between $\mathrm{ACPA}^{+}$and $\mathrm{ACPA}^{-}$RA patients was observed. Interestingly, sorted B cells from healthy subjects showed increased sensitivity to in vitro stimulation with increased expression of CD80 and CD86 when cultured under hypoxic conditions, while co-culture with RA patient synovial fibroblasts did not enhance this effect.

Conclusions: The increased capacity of $\mathrm{ACPA}^{+}$compared to ACPA- RA patient synovial $C D 4^{+} T$ cells to produce pro-inflammatory cytokines, could be responsible for the more severe disease progression of $\mathrm{ACPA}^{+}$compared to ACPA RA. The accumulation of memory $B$ cells in both $\mathrm{ACPA}^{+}$and $\mathrm{ACPA}^{-} \mathrm{RA}$, underlines a common, antibody independent, contribution of $B$ cells in synovial inflammation. While $B$ cell activation under hypoxic conditions and increased CD80/CD86 expression is potentially an important mediator for the emergence of auto-reactive $T$ cells and disease progression in RA.

Disclosure of Interest: None declared

DOI: 10.1136/annrheumdis-2018-eular.4835

\section{OP0315 JUVENILE IDIOPATHIC ARTHRITIS PATIENTS EXHIBIT PERSISTENCE IN CD4 MEMORY T CELLS AND DISTINCT TRANSCRIPTOMIC SIGNATURE DESPITE BIOLOGICS THERAPY}

J.Y. Leong ${ }^{1}$, J.G. Yeo ${ }^{1}$, P. Chen ${ }^{1}$, F. Ally ${ }^{1}$, C. Chua ${ }^{1}$, S.N. Hazirah ${ }^{1}$, P. Lu ${ }^{1}$, L. Lai ${ }^{1}$, L.D.T. Bathi ${ }^{1}$, T. Arkachaisri ${ }^{1,2}$, D.J. Lovell ${ }^{3,4}$, S. Albani ${ }^{5} .{ }^{1}$ Translational Immunology Institute, Singapore Health Services Pte Ltd, Singhealth/Duke-NUS Academic Medical Centre; ${ }^{2} K K$ Women's and Children's Hospital, Singapore Health Services Pte LtD, Singapore, Singapore; ${ }^{3}$ Division of Rheumatology, Cincinnati Children's Hospital Medical Center, ${ }^{4}$ Department of Paediatrics, University of Cincinnati College of Medicine, Cincinnati, USA; ${ }^{5}$ Translational Immunology Institute, Singapore Health Services Pte Ltd, Singhealth/Duke-NUS Academic Medical, Singapore, Singapore

Background: JIA patients respond well to anti-TNFA biologics, with up to $80 \%$ of patients achieving clinical remission. In spite of this success, $50 \%-80 \%$ will relapse upon therapy withdrawal, indicating a large proportion of patients had yet to fully resolve their disease. Compounded by concerns with drug toxicities and financial burden, there is a genuine interest to find predictors for successful drug cessation and devise new avenues of therapy.
Objectives: To determine how subclinical persistence of disease occurs despite therapy, we compare JIA individuals destined to flare or remain inactive, prior to (To) and after therapy withdrawal (Tend). Previous publications have reveal that CD4 T cells play a vital role in disease pathogenesis in JIA patients. We aim to dissect the CD4 landscape (a) through CyToF, to decipher the CD4 subsets responsible for disease persistence, (b) to unravel the pathways involved through mRNA analysis with Nanostring.

Methods: Patients treated with anti-TNF-alpha biologics were recruited with clinically inactive disease on treatment and initiated with therapy discontinuation. The patients designated as flare $(n=24)$ and inactive $(n=24)$ based on 6 JIA core set parameters. Healthy paediatric controls $(n=17)$ with no inflammatory disease were recruited pre-operatively during day surgeries. A separate study with active JIA patients recruited pre/post treatment $(n=4$ paired) with anti-TNFA biologics and achieving recent clinical remission.

Results: Interrogation of PBMCs with CyToF reveal the persistence of a subset of CD3 +CD4+inflammatory memory CD45RA- TNFA +PD1- CTLA4- T cells $(\mathrm{p}<0.05)$ in flare $(\mathrm{To})$ versus inactive $(\mathrm{To})$ individuals. Intriguingly an additional subclinical subset, TNFA +IL-6+, was detected $(p<0.05)$ in flare $(T o)$ versus healthy individuals. Upon therapy withdrawal, this subclinical subset expands $(p<0.05)$ in flare $(T e n d)$ individuals versus inactive (Tend). Notably we also observe a distinct early increase in CD3 +CD4+CD45 RA- CXCR5 + T cells in flare versus inactive (To) individuals which subsides after therapy withdrawal, indicating early $\mathrm{T}-\mathrm{B}$ interaction. We noted strong but likely inadequate compensatory enrichment of CD45RA- subset of Tregs in flare versus inactive (To/Tend) individuals. To decipher the mechanism that leads to incomplete disease resolution, we sorted CD3 +CD4+CD45 RA- CD45RO+T cells from flare and inactive (To/Tend) patients, and observed striking dysregulation in several major pathways, (a) TCR activation, (b) TNFA signalling, (c) Apoptosis, (d) NF-kB signalling, (e) MAPK signalling. This dysregulation also extends to a separate cohort of active JIA patients naive to anti-TNFA biologics therapy and persisting till recent onset clinical remission.

Conclusions: These results highlight a strong immunological memory dysregulation in a subset of CD4 T cells in JIA patients that is predictive of clinical fate and providing new therapeutic insights.

Disclosure of Interest: None declared

DOI: 10.1136/annrheumdis-2018-eular.5194

\begin{tabular}{l|l}
\hline OP0316 & INCREASED EXPRESSION OF MICRORNA-142-3P IS \\
ASSOCIATED WITH THE FUNCTIONAL DEFECT OF \\
REGULATORY T CELLS IN ANTI-NEUTROPHIL \\
CYTOPLASMIC ANTIBODY ASSOCIATED VASCULITIS
\end{tabular}

G. Dekkema ${ }^{1 *}$, T. Bijma ${ }^{1,2}$, W. Abdulahad ${ }^{3}$, P. Jellema ${ }^{1}$, A. Van Den Berg ${ }^{1}$, B. J. Kroesen ${ }^{4}$, C. Stegeman ${ }^{2}$, P. Heeringa ${ }^{1}$, J.-S. Sanders ${ }^{2} .{ }^{1}$ Pathology and Medical Biology; ${ }^{2}$ Department of Internal Medicine, division of Nephrology; ${ }^{3}$ Department of Rheumatology and Clinical Immunology, ${ }^{4}$ Department of Clinical Immunology, University Medical Center Groningen, Groningen, Netherlands

Background: Circulating regulatory T cells (Tregs) in anti-neutrophil cytoplasmic antibody associated vasculitis (AAV) are frequently functionally deficient. The mechanism behind their impaired function is however unknown. Small non-coding microRNA (miR) are post-transcriptional regulators of protein synthesis and previous studies have shown that differently expressed miRs in T cells are associated with auto immunity.

Objectives: To investigate whether the dysfunctionality of Tregs in AAV is due to altered microRNA ( $\mathrm{miR}$ ) expression.

Methods: Tregs $\left(\mathrm{CD} 4^{+} \mathrm{CD} 45 \mathrm{RO}^{+} \mathrm{CD} 25^{+} \mathrm{CD} 127\right)$ of healthy controls $(\mathrm{HC})$ and AAV patients in remission without treatment (AAV-REM) were FACS-sorted, and total RNA was isolated. Samples from $8 \mathrm{HCs}$ and $8 \mathrm{AAV}$-REMs were subjected to miRNA microarray analysis. Based on relative expression and fold change, 5 differentially expressed miRs were validated in an independent cohort using qRTPCR and a database and literature search was performed to identify potential targets.

Results: Nineteen miRs differentially expressed were detected by microarray analysis, of which Let-7g, miR-20a-5p, miR-26a-5p, miR-142-3 p, miR-146a-5p were validated in an independent cohort. Of these, miR-142-3 $p$ was confirmed to be significantly upregulated (2-fold, $p=0.03$ ) in Tregs from AAV-REM patients compared to HC Tregs $(n=23, n=22)$. To study the functional impact of miR-142$3 p$ overexpression, HC Tregs were transfected using either a mimic-miR-142$3 p$ or a scrambled (SCR)-control. After transfection, live Tregs were co-cultured with $\mathrm{T}$ effectors $\left(\mathrm{CD} 4^{+} \mathrm{CD} 25^{-}\right)$in a suppression assay to test their suppressive capacity. Transfection with mimic-miR-142-3 $p$ significantly increased the miR $142-3$ p levels ( 2.4 fold, $p=0.03$ ) and reduced the suppressive capacity compared to SCR-transduced Tregs (1.9 fold reduction, $p=0.02$ ). Moreover, miR-142-3 $p$ levels tended to correlate to the suppressive function of Tregs $(p=0.06$, rho $=-0.591)$. A database and literature search identified adenylyl cyclase 9 (AC9) as a promising target of miR-142-3 p. mRNA levels of AC9 tended to be lower in AAV-REM patients compared to $\mathrm{HC}$ ( 3.8 fold, $\mathrm{p}=0.07$ ). In addition, cAMP 
levels, which are partly produced by AC9, were significantly lower in Tregs from AAV-REM patients after 48 hour of stimulation with anti-CD3 and anti-CD28 (1.7 fold, $p=0.003$ ).

Conclusions: Increased expression of miR-142-3 $p$ in Tregs of AAV-REM patients is associated with their functional impairment, potentially by targeting the AC9/CAMP mediated suppression.

Disclosure of Interest: G. Dekkema: None declared, T. Bijma: None declared, W. Abdulahad Grant/research support from: European Union's Horizon 2020 research and innovation program under grant No 668 036., P. Jellema: None declared, A. Van Den Berg: None declared, B. J. Kroesen: None declared, C. Stegeman: None declared, P. Heeringa Grant/research support from: European Union's Horizon 2020 research and innovation program under grant No 668 036., J.-S. Sanders Grant/research support from: Nierfonds grant no. $130 K J 39$

DOI: 10.1136/annrheumdis-2018-eular.3226

\section{OP0317 SCREENING OF AN AUTOANTIBODY SIGNATURE OF EARLY KNEE OSTEOARTHRITIS: DATA FROM THE OSTEOARTHRITIS INITIATIVE}

M. Camacho Encina ${ }^{1 *}$, V. Calamia ${ }^{1}$, F. Picchi ${ }^{1}$, J. VanDuin ${ }^{2}$, J. Qiu ${ }^{2}$, C. Ruiz Romero', J. LaBaer ${ }^{2}$, F.J. Blanco' ${ }^{1}{ }^{1}$ Rheumatology Research Group, ProteoRed, PRB2-ISCIII, INIBIC-Complejo Hospitalario Universitario A Coruña, A Coruña, Spain; ${ }^{2}$ Virginia G. Piper Center for Personalized Diagnostics, Biodesign Institute, Arizona State University, Tempe, Arizona, USA

Background: The immune system can detect the changes involved in the osteoarthitic $(\mathrm{OA})$ joint, triggering the production of immunoglobulins against self-proteins (Autoantibodies or AAbs). As AAbs might be generated in a stage prior to the disease, they can be potentially used to identify an increased risk for the disorder, allowing the diagnosis of asymptomatic OA

Objectives: The discovery of an AAb signature useful for the early diagnosis of knee OA.

Methods: Nucleic Acid-Programmable Protein Arrays (NAPPA) were used to screen the presence of AAbs against 2200 human proteins in sera belonging to two different subcohorts from the Osteoarthritis Initiative (OAI): Incidence and Non-exposed subcohorts. Participants in the incidence subcohort had not developed any symptomatic knee OA at baseline, but they did present an increased risk of developing the disease. Non-exposed subcohort incorporate participants which have no radiographic findings or symptoms of knee OA, nor any reported risk factors. A hundred sera from both subcohorts at baseline were used. In order to know if the profile of AAbs was specific of asymptomatic OA, samples belonging to the same patients selected from the incidence subcohort were analysed after 72 months of follow up, when all of them have developed OA. Data were normalised following the Biodesing Institute criteria. A 1.1 cutoff was used to determine reactivity and all proteins over the cutoff were analysed by Wilcoxon test. The Partial Area Under the Curve (pAUC) at 95\% specificity was analysed with a $\mathrm{p}$ value $<0.05$

Results: We detect AAbs against six proteins showing different reactivity (see table 1) in a stage prior to the disease -samples from the incidence subcohort at baseline compared with the non-exposed subcohort-. These proteins were implicated in the colesterol biosynthesis (Diphosphomevalonate decarboxylase, $M V D$ ), and the elimination of pottentially toxic xenobiotic or endogenous compounds (ASB7 and UGT1A7, repectively). We also found a GTPase from the Rho family (RAC3), the Vacuolar protein sorting-associated protein 4B (VPS4B), which has been recently reported to facilitate chondrocyte apoptosis in a OA rat model, and Methionine adenosyltransferase 2 subunit beta (MAT2B). The latter is the regulatory subunit of the enzyme responsible of the catalysis of S-adenosylmethionine, a dietary supplement widely used in the management of OA symptoms. None of the six AAbs were found after the disease has been established (samples from the incidence subcohort at 72 months).

\begin{tabular}{|c|c|c|c|c|c|}
\hline $\begin{array}{l}\text { Acc } \\
\text { number }\end{array}$ & Symbol & $\begin{array}{l}\text { Wilcoxon test } \\
\text { (pvalue) }\end{array}$ & $\begin{array}{c}\text { Specificity at } \\
95 \%\end{array}$ & AUC & $\begin{array}{c}\text { AUC ( } p \\
\text { value) }\end{array}$ \\
\hline 075351 & VPS4B & 0.005 & $80.80 \%$ & 0.855 & 0.4732 \\
\hline Q9NZL9 & МAT2B & 0.005 & $80.80 \%$ & 0.86 & 0.0497 \\
\hline P53602 & MVD & 0.002 & $75.60 \%$ & 0.9 & 0.0426 \\
\hline$P 60763$ & RAC3 & 0.003 & $76.30 \%$ & 0.88 & 0.0496 \\
\hline Q9HAW7 & UGT1A7 & 0.015 & $79.59 \%$ & 0.82 & 0.0481 \\
\hline Q9H672 & ASB7 & 0.002 & $76.90 \%$ & 0.885 & 0.0499 \\
\hline
\end{tabular}

Conclusions: This work is the first to screen a large number of human proteins for the discovery of OA-associated AAbs. We define a panel of six AAbs, which are increased prior to the development of symptomatic knee OA. These results suggest that a serum AAbs signature can facilitate the discovery of early OA biomarkers useful for clinical diagnosis.

Acknowledgements: This work has received financial support from the Xunta de Galicia and the European Union (European Social Fund - ESF).

Disclosure of Interest: None declared

DOI: 10.1136/annrheumdis-2018-eular.4522

\section{OP0318 NETOSIS-INHIBITING T-ACPA THERAPY FOR USE IN DIFFERENT NET-DRIVEN HUMAN AUTOIMMUNE DISEASES}

R.G. Chirivi ${ }^{1 *}$, J.W. van Rosmalen ${ }^{2}$, K. Kambas $^{3}$, G. Schmets $^{2}$, H. Kalisvaart ${ }^{2}$ G. Bogatkevich ${ }^{4}$, T. Shaw ${ }^{5}, \mathrm{H}$. van $\mathrm{Es}^{5}$, J.M. Raats ${ }^{1} .{ }^{1}$ Citryll and ModiQuest BV; ${ }^{2}$ ModiQuest BV, Oss, Netherlands; ${ }^{3}$ Laboratory of Molecular Hematology, Democritus University of Thrace, Alexandroupolis, Greece; ${ }^{4}$ Department of Medicine, Medical University of South Carolina, Charleston, USA $;{ }^{5}$ Citryll BV, Oss, Netherlands

Background: Aberrant Neutrophil Extracellular Trap (NET) formation contributes to the induction and propagation of inflammation and plays a key role in causing tissue damage in conditions like sepsis, SLE, RA and vasculitis. Citrullination of proteins is involved in the formation of NETs, autoimmunity, and the breaking of tolerance in NET-driven autoimmune diseases. In SLE and RA, neutrophils undergo enhanced NETosis, and NET components are observed in blood, inflamed tissues and joints.

Objectives: Our objective is to develop a novel first in class NET-inhibiting therapeutic anti-citrullinated protein antibody (tACPA) targeting citrullinated histones $2 \mathrm{~A}$ and 4 , for the treatment of human diseases in which aberrant NET formation add to the severity of the pathology with an initial focus on autoimmune diseases. Here, we demonstrate the utility of tACPA for different NET-based diseases beyond RA, including SLE, vasculitis, gout and idiopathic pulmonary fibrosis (IPF)

Methods: Previously, using two RA animal models, the therapeutic properties of

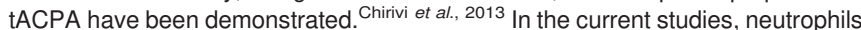
from RA and SLE donors, as well as biological NET-inducing stimuli, such as RA synovial fluid (SF), gout SF and activated platelets, have been used to demonstrate the NETosis-inhibiting properties of tACPA in different human disease con texts. We have further expanded tACPA's therapeutic utility by testing it in a surrogate model for NET-mediated organ damage (sepsis) and IPF.

Results: NETosis in human RA and SLE neutrophils have been induced with a calcium ionophore and could be inhibited by tACPA treatment $(40 \%-100 \%$ reduction). Similar results were obtained using RA and gout SF or activated platelets as NETosis inducers in combination with neutrophils from healthy donors. These observations have been confirmed with multiple NET readouts such as MPO activity, MPO/DNA ELISA, DNA quantification as well as imaging readouts. In addition, we demonstrated that in an LPS-induced sepsis model $30 \%$ of tACPA treated mice survived (compared to $0 \%$ in placebo controls), showing protection against organ failure. In a bleomycin-induced IPF mouse model, tACPA protected mice from the development of lung fibrosis (compared to placebo controls). When determining neutrophil counts in bronchoalveolar lavage samples, we found that in tACPA-treated mice, neutrophil levels were normal, while levels in placebotreated mice were elevated.

Conclusions: In a sepsis and IPF mouse model, tACPA prevented NET-mediated organ damage, providing evidence that tACPA could be a promising therapeutic strategy for diseases where NET-mediated endothelial toxicity causes organ damage like SLE, vasculitis and IPF. Central to our strategy for generating a preclinical data package supporting clinical testing, is to demonstrate that patient NETosis can be significantly inhibited ex vivo. We will present data that confirm that TACPA can block human SLE NETosis as well as human NETosis induced by activated platelets or gout SF.

Disclosure of Interest: R. Chirivi Shareholder of: Citryll BV, Employee of: ModiQuest BV, J. van Rosmalen Employee of: ModiQuest BV, K. Kambas: None declared, G. Schmets: None declared, H. Kalisvaart: None declared, G. Bogatkevich: None declared, T. Shaw: None declared, H. van Es Shareholder of: Citryll BV, J. Raats Shareholder of: Citryll BV

DOI: 10.1136/annrheumdis-2018-eular.1607 